\title{
DISC 2011 Invited Lecture: Polygon Reconstruction with Little Information: An Example for the Power of Simple Micro-robots
}

\author{
Peter Widmayer \\ Department of Computer Science, ETH Zurich, Zurich, Switzerland
}

\begin{abstract}
For which settings will local observations of an unknown environment allow simple mobile agents (micro-robots) to draw global conclusions? This question has been studied for a long time when the environment is a graph, and the mobile agents walk on its vertices, under a variety of models. In this talk, however, we are interested in geometric environments.

More specifically, we discuss the problem of reconstructing an unknown simple polygon from a series of local observations. We aim to understand what types of sensing information acquired at vertices of the polygon carry enough information to allow polygon reconstruction by mobile agents that move from vertex to vertex. It turns out that ideas from distributed computing help to reconstruct the polygon topology even if the sensing information is purely non-geometric. We also briefly touch on a few related problems such as guarding the polygon and rendezvous. The reported work has been done over the years with Davide Bilo, Jeremie Chalopin, Shantanu Das, Yann Disser, Beat Gfeller, Matus Mihalak, Subhash Suri, and Elias Vicari.
\end{abstract}

